\title{
Stagonospora avenae Secretes Multiple Enzymes that Hydrolyze Oat Leaf Saponins
}

\author{
John P. Morrissey, Jos P. Wubben, and Anne E. Osbourn \\ The Sainsbury Laboratory, John Innes Centre, Norwich Research Park, Norwich NR4 7UH, U.K. \\ Accepted 5 July 2000.
}

\begin{abstract}
The phytopathogenic fungus Stagonospora avenae is able to infect oat leaves despite the presence of avenacoside saponins in the leaf tissue. In response to pathogen attack, avenacosides are converted into 26-desglucoavenacosides (26-DGAs), which possess antifungal activity. These molecules are comprised of a steroidal backbone linked to a branched sugar chain consisting of one $\alpha$-L-rhamnose and two (avenacoside A) or three (avenacoside B) $\beta$-D-glucose residues. Isolates of the fungus that are pathogenic to oats are capable of sequential hydrolysis of the sugar residues from the 26-DGAs. Degradation is initiated by removal of the L-rhamnose, which abolishes antifungal activity. The D-glucose residues are then hydrolyzed by $\beta$-glucosidase activity. A comprehensive analysis of saponin-hydrolyzing activities was undertaken, and it was established that $S$. avenae isolate WAC1293 secretes three enzymes, one $\alpha$ rhamnosidase and two $\beta$-glucosidases, that carry out this hydrolysis. The major $\beta$-glucosidase was purified and the gene encoding the enzyme cloned. The protein is similar to saponin-hydrolyzing enzymes produced by three other phytopathogenic fungi, Gaeumannomyces graminis, Septoria lycopersici, and Botrytis cinerea, and is a family $3 \beta$-glucosidase. The gene encoding the $\beta$-glucosidase is expressed during infection of oat leaves but is not essential for pathogenicity.
\end{abstract}

Additional keywords: fungal pathogen, saponinase, secondary metabolite.

The production of low-molecular-weight antimicrobial molecules is one component of plant defense against pathogens. Some of these defense molecules (for example, phytoalexins and antimicrobial peptides) are induced in response to pathogen attack, whereas others, such as the saponins, are present constitutively in plant tissue. Saponins are glycosylated triterpenoid, steroid, or steroidal alkaloid molecules found in a large number of plants. A variety of biological roles have been pos-

Corresponding author: Anne E. Osbourn;

E-mail: annie.osbourn@bbsrc.ac.uk

Current address of John P. Morrissey: Microbiology Department, University College Cork, Ireland.

Current address of Jos P. Wubben: PBG Aalsmeer, Linnaeuslaan 2a, 1431 JV Aalsmeer, the Netherlands.

Genomic sequence data has been deposited in the EMBL database under accession number AJ276675. tulated for different saponins, involving allelopathic activity (Fukuhara and Kubo 1991; Oleszek and Jurzysta 1987), defense against insects or herbivores (Weissenberg et al. 1998), and protection against fungal pathogens (Osbourn 1996). A number of saponins have been shown to inhibit fungal growth in culture and, because these secondary metabolites are generally concentrated in peripheral plant tissues, it has been proposed that they may form a first line of defense against infection (Osbourn 1996; VanEtten et al. 1995). The mechanism of antifungal action of saponins is not well understood but it is believed that they complex with sterols in the cell membrane, leading to pore formation and consequent loss of membrane integrity (Armah et al. 1999; Keukens et al. 1992, 1995).

Oats are unusual among plants in that they synthesize two distinct classes of saponins: triterpenoid avenacins in the roots and steroidal avenacosides in the leaves (Osbourn 1996). Avenacins are a family of four related saponins, all possessing an identical branched sugar chain at the $\mathrm{C}-3$ position, but differing in modifications to the triterpenoid backbone. The sugar chain is important for the antifungal activity of avenacins, and removal of a single terminal D-glucose residue is sufficient to dramatically reduce their toxicity (Osbourn et al. 1991). Mutant oat lines that had reduced levels of avenacins displayed an increased susceptibility to a number of fungal pathogens, consistent with the premise that avenacins protect oat roots against infection (Papadopoulou et al. 1999). Some fungi, however, can infect roots despite the presence of avenacins and, in some cases, this could involve avoidance, tolerance, or neutralization of the saponins. The importance of saponin detoxification has been studied for the root-infecting pathogen Gaeumannomyces graminis. This fungus secretes a $\beta$-glucosidase that removes terminal D-glucose residues from the avenacin sugar chain, converting the saponins to less toxic molecules (Osbourn et al. 1991). A mutant strain of $G$. graminis lacking the saponin-hydrolyzing $\beta$-glucosidase (avenacinase) could no longer infect oats but was still able to infect cereals that did not contain saponins, providing evidence that the avenacinase enzyme is specifically required for infection of oats (Bowyer et al. 1995). Taken together, the data from the oat mutants lacking avenacin and from the fungal mutant lacking avenacinase show that avenacin acts as a phytoprotectant and that production of avenacinase by $G$. graminis is an important host range determinant.

The second family of oat saponins are the steroidal avenacosides A and B, present in the leaves of the plant. Avenacoside $\mathrm{B}$ differs from avenacoside $\mathrm{A}$ only in that it pos- 
sesses an additional D-glucose residue in the branched sugar chain attached to the C-3 position of the backbone (Fig. 1). Avenacosides are stored in an inactive form in the vacuoles of epidermal cells (Kesselmeier and Urban 1983; Urban et al. 1983) and are converted by a plant enzyme into active 26-desglucoavenacosides (26-DGAs) in response to attack or wounding (Fig. 1) (Gus-Mayer et al. 1994). It was previously reported that 26-DGAs have membraneolytic activity against a range of fungi but that two oat leaf-infecting species, Stagonospora avenae (Frank) Bisset (syn. Septoria avenae) and Drechslera avenacea, are resistant to this activity (Lüning and Schlösser 1976). These two foliar pathogens of oats are capable of enzymatic degradation of 26-DGAs. Hydrolysis products of 26-DGAs were detected in leaves infected with $D$. avenacea, consistent with the enzymatic removal of sugars by the fungus (Lüning and Schlösser 1976). More recently, it was demonstrated that oat-attacking isolates of $S$. avenae were resistant to 26-DGAs in culture and able to detoxify the saponins (Wubben et al. 1996). An analysis of the avenacoside-hydrolyzing activities of one $S$. avenae strain (isolate WAC1293) was carried out (Wubben et al. 1996). When 26-DGA-B was incubated with $S$. avenae culture filtrate, the sugars were hydrolyzed in a sequential fashion (Fig. 1). The initial step of the hydrolysis is removal of the terminal L-rhamnose to produce species IV. The antifungal activity of this molecule is considerably less than that of 26-DGA (Wubben et al. 1996); therefore, L-rhamnose removal can be considered a detoxification reaction. The purification of an avenacosidase activity that carried out both this reaction and the subsequent hydrolysis of Dglucose residues was described, and it was suggested that a single enzyme might possess both $\alpha$-rhamnosidase and $\beta$-glucosidase activity (Wubben et al. 1996). This article reports the development of refined assays and protein fractionation to characterize the avenacoside-hydrolyzing enzymes secreted by $S$. avenae isolate WAC1293. It was established that this fungus synthesizes multiple enzymes that hydrolyze the avenacoside sugar chain. A single $\alpha$ rhamnosidase that is capable of carrying out the detoxification of 26-DGAs is produced. In addition, two independent $\beta$-glucosidases that subsequently hydrolyze D-glucose residues from the saponins were identified. The gene encoding the major $\beta$-glucosidase was cloned, and a mutant lacking this enzyme was constructed to investigate a possible role for this enzyme in pathogenicity.

\section{RESULTS}

Separation of three avenacoside-hydrolyzing enzymes.

It was previously reported that incubation of avenacosides, or 26-DGAs, with $S$. avenae culture filtrate resulted in the sequential hydrolysis of the L-rhamnose and D-glucose sugar residues from the saponins (Wubben et al. 1996). To ascertain whether a single enzyme possessed both $\alpha$-rhamnosidase and $\beta$-glucosidase activity or whether more than one enzyme was involved, we undertook a comprehensive analysis of avenacoside-hydrolyzing activities produced by $S$. avenae isolate WAC1293. Proteins were concentrated from culture filtrate and fractionated by anion-exchange chromatography. Fractions were then assayed for $\alpha$-rhamnosidase and $\beta$-glucosidase activity toward 26-DGAs (Fig. 2). Removal of the L-rhamnose residue from 26-DGA-B yields de-rhamnosyl-26-DGA-B (Fig. 1 , species IV), which is less polar and can be separated from 26-DGA-B by thin-layer chromatography (TLC). The product of the $\alpha$-rhamnosidase is a substrate for $\beta$-glucosidase activity; therefore, an inhibitor of $\beta$-glucosidase enzymes was included in the reactions. This blocked the further hydrolysis of de-rhamnosyl-26-DGA-B and facilitated the detection of a single $\alpha$-rhamnosidase peak in fractions 7 to 9 (Fig. 2A). To assay for $\beta$-glucosidase activity, the same fractions were incubated with de-rhamnosyl-26-DGA-B. This substrate was used because anal-

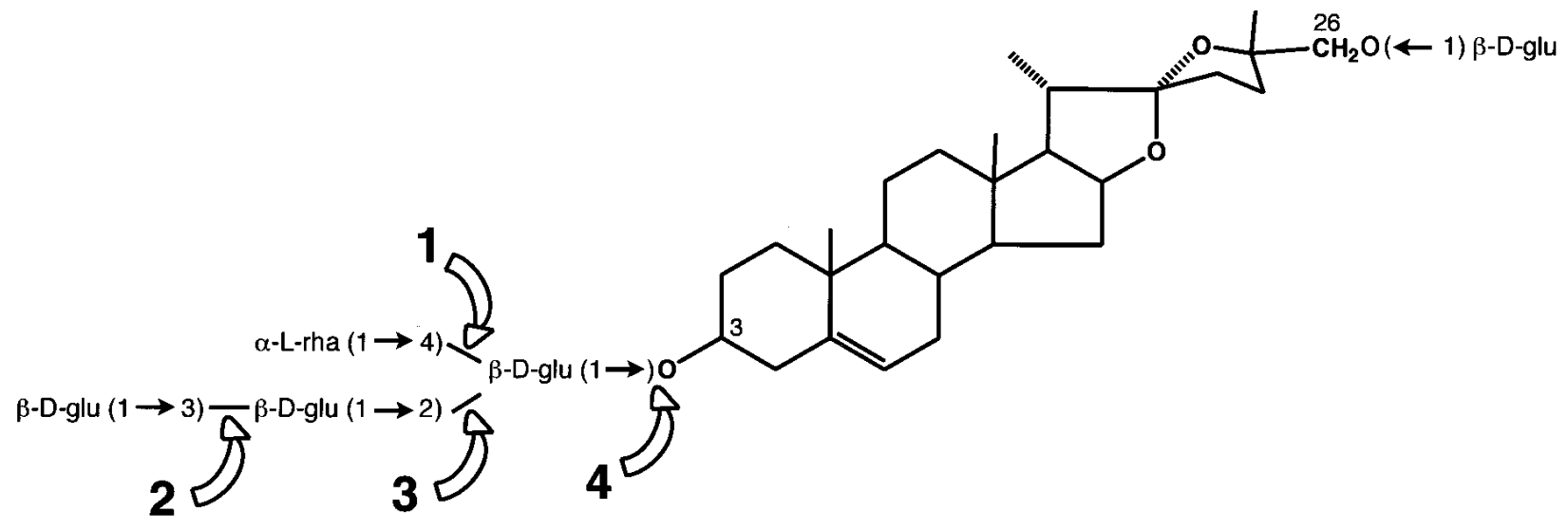

Fig. 1. Hydrolysis of avenacoside B by oat and Stagonospora avenae enzymes. Avenacosides A and B are composed of a steroidal backbone with a Dglucose attached to the C-26 position and a branched sugar chain linked to the C-3 carbon. Avenacoside B contains four sugar residues in this chain, whereas avenacoside A lacks the terminal D-glucose residue and contains three sugars. In response to attack or damage, a plant enzyme converts avenacosides to the active molecules 26-desglucoavenacosides by the removal of the C-26 D-glucose residue. Enzymes secreted by $S$. avenae hydrolyze sugar residues from the C-3 branched sugar chain. The sites of hydrolysis are shown, with the order of cleavage indicated. Following established nomenclature (Wubben et al. 1996), cleavage 1 by $\alpha$-rhamnosidase activity produces species IV, and cleavages 2,3 , and 4 by $\beta$-glucosidase activity give rise to species III, II, and I, respectively. 
ysis of the kinetics of hydrolysis has indicated that efficient $\beta$-glucosidase activity requires the prior removal of the terminal L-rhamnose residue from avenacosides. Two distinct $\beta$-glucosidase activities were detected, peaking in fractions 3 to 5 and fractions 10 to 12 (Fig. 2B). We designate the $\beta$-glucosidase enzyme in fraction 10 to 12 as $S$. avenae $\beta$-glucosidase 1 (savBGL1) and that in fractions 3 to 5 as $S$. avenae $\beta$-glucosidase 2 (savBGL2). Under the conditions employed in this assay, savBGL1 (Fig. 2, lanes 10-12) produces species III, II, and I (Fig. 1) from the substrate, species IV. These molecules arise from the sequential removal of D-glucose residues and, on longer incubation, savBGL1 converts all the starting substrate to species I, the aglycone (data not shown). In contrast, savBGL2 mainly yields species III from this substrate (Fig. 2B, lanes 3-5). The savBGL2 enzyme further hydrolyzes species III to species II (Fig. 2B, lanes 3-5) and, on longer incubation, some of the saponin is hydrolyzed completely to produce species I (data not shown), but the efficiency is low compared with savBGL1. Based on these data, savBGL1 can be considered the major avenacosidehydrolyzing $\beta$-glucosidase produced under these culture conditions. The clear separation of the three peaks of activity demonstrates unequivocally that different enzymes are responsible for the removal of L-rhamnose and Dglucose residues from avenacosides and that two independent $\beta$-glucosidases that can utilize avenacosides as a substrate are produced by this fungal isolate.

\section{Isolation of the gene encoding savBGL1.}

Previously, partial purification of avenacosidase-hydrolyzing activity from S. avenae isolate WAC1293 generated an enriched protein preparation from culture filtrate that possessed both $\alpha$-rhamnosidase and $\beta$-glucosidase activ- ities (Wubben et al. 1996). This protein preparation was fractionated using the conditions described for Figure 2, and it was determined that the major protein in the preparation was savBGL1 and that only trace amounts of an $\alpha$ rhamnosidase were present (data not shown). The enriched savBGL1 protein preparation was digested with cyanogen bromide, and two resulting peptides were sequenced. Peptide 1, AAFYYVGRDKARIPVNFD, and peptide 2, GWGSTSDYPYLVTPLES, were used to search the SwissProt database using the BLAST program, and it was found that both peptides bore homology to family $3 \beta$-glucosidases. This information, along with the experimental data described above (Fig. 2B), allowed the prediction that savBGL1 was a family $3 \beta$-glucosidase. Proteins in this family have regions of high sequence conservation; therefore, the relative positions of the peptides within the protein could be assigned. The peptide sequences were used to design two degenerate polymerase chain reaction (PCR) primers, SAV01 (forward) and SAV02 (reverse). Two further degenerate primers were designed based on the sequence of conserved regions of family $3 \beta$-glucosidases. These primers, F3/1 and F3/3, were chosen to be upstream and downstream of SAV01 and SAV02, respectively, allowing the use of a nested PCR strategy to amplify a segment of the gene. Nested PCR on $S$. avenae genomic DNA using F3/1-F3/3 followed by SAV01-SAV02 amplified a 350-base pair (bp) fragment, the deduced amino acid sequence of which contained both peptide sequences. PCR using the combination F3/1-SAV02 amplified an overlapping 950-bp fragment.

The 950-bp DNA fragment was used as a probe to screen a genomic library of isolate WAC1293 constructed in the $\lambda$-ZAPII phagemid system. Screening of 150,000 recombinant plaques resulted in the isolation of a number of

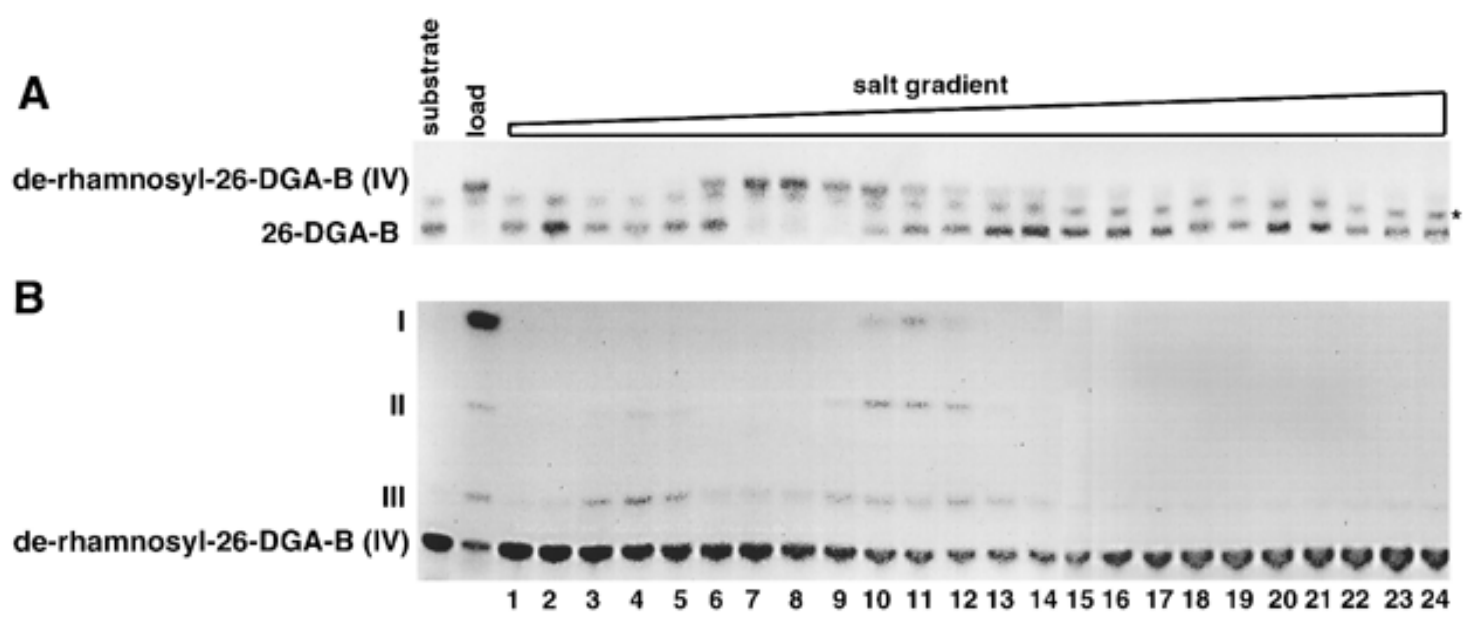

Fig. 2. Stagonospora avenae secretes multiple avenacoside-hydrolyzing enzymes. Proteins were concentrated from $S$. avenae culture filtrate, bound to an HQ anion-exchange column, and eluted with a salt gradient. Each fraction was then assayed for avenacoside-hydrolyzing activities. Following incubation of the substrate with an aliquot of each fraction, the reactions were loaded onto silica plates and substrates and products were resolved by thin-layer chromatography. A, To detect $\alpha$-rhamnosidase activity, molecule 26 -desglucoavenacoside (26-DGA)-B was incubated with aliquots from each fraction in the presence of an inhibitor of $\beta$-glucosidase activity (deoxynojirimycin). As controls, 26-DGA-B and the result of incubation of this substrate with total filtrate protein (load) are shown. There is a minor contaminant, indicated on the right with an asterisk, present in the substrate preparation. B, To detect $\beta$-glucosidase activity, species IV, the product of hydrolysis of 26-DGA-B by the $\alpha$-rhamnosidase, was used as a substrate and incubated with proteins from the same fractions. The products of sequential hydrolysis of species IV by $\beta$-glucosidases are indicated on the left. The structures of IV, III, II, and I are illustrated in Figure 1. 
overlapping phagemids. Based on the position of the hybridizing DNA, one phagemid was selected and the insert was sequenced completely (EMBL accession number AJ276675). The sequence analysis predicted that a single open reading frame (ORF) separated by three introns of 165,175 , and 48 nucleotides (nt) was present. The ORF starts at position 2,944 of the sequenced insert and ends at position 5,948. The introns are located at positions 2,992 to $3,157,3,244$ to 3,419 , and 3,790 to 3,838 . Reversetranscriptase (RT)-PCR analysis confirmed the presence and locations of the three introns (data not shown). The gene is predicted to encode a protein of 871 amino acids (aa), with a calculated molecular mass of $94 \mathrm{kDa}$ and an isoelectric point of 4.6. A 14-aa putative secretion signal sequence is located at the $\mathrm{N}$-terminus of the proteins. The deduced amino acid sequence and an alignment against two representative members of family $3 \beta$-glucosidases, avenacinase from $G$. graminis and trBGL1 from Trichoderma reesei, are shown in Figure 3. The savBGL1 enzyme is homologous over the length of the protein to other family 3 fungal $\beta$-glucosidases and contains the two conserved amino acids that are believed to be the catalytic residues of these enzymes, aspartate at position 291 and glutamate at position 519 (Varghese et al. 1999). The protein also contains an additional domain, located approxi- mately between residues 675 and 760 , that is absent from the majority of enzymes in this group.

\section{Comparison of family 3 saponin-hydrolyzing enzymes.}

Family $3 \beta$-glycosidases constitute a large protein family. The complete sequences of more than 60 predicted members are now present in the databases, and more are regularly being identified in genome-sequencing projects. A phenogram shows the relatedness between the predicted amino acid sequences of the characterized eukaryotic family $3 \beta$-glycosidases (Fig. 4). It is seen that fungal $\beta$ xylosidases, plant $\beta$-glucosidases, and fungal $\beta$-glucosidases form distinct subgroups. Enzymes in this family act on a range of substrates, including cellulose (Lymar et al. 1995), plant glucans (Hrmova and Fincher 1997), and saponins. Three enzymes that are known to hydrolyze sugars from saponins are present in the fungal $\beta$-glucosidase subgroup. These are avenacinase from $G$. graminis (U35463) (Bowyer et al. 1995) and Sap1 from Botrytis cinerea (AJ130890) (Quidde et al. 1999), which act on the triterpenoid avenacins, and tomatinase from Septoria lycopersici (U24701), which degrades the steroidal alkaloid $\alpha$ tomatine (Osbourn et al. 1995; Sandrock et al. 1995). In each case, the enzyme removes a terminal D-glucose residue, but the saponin backbones are structurally distinct.

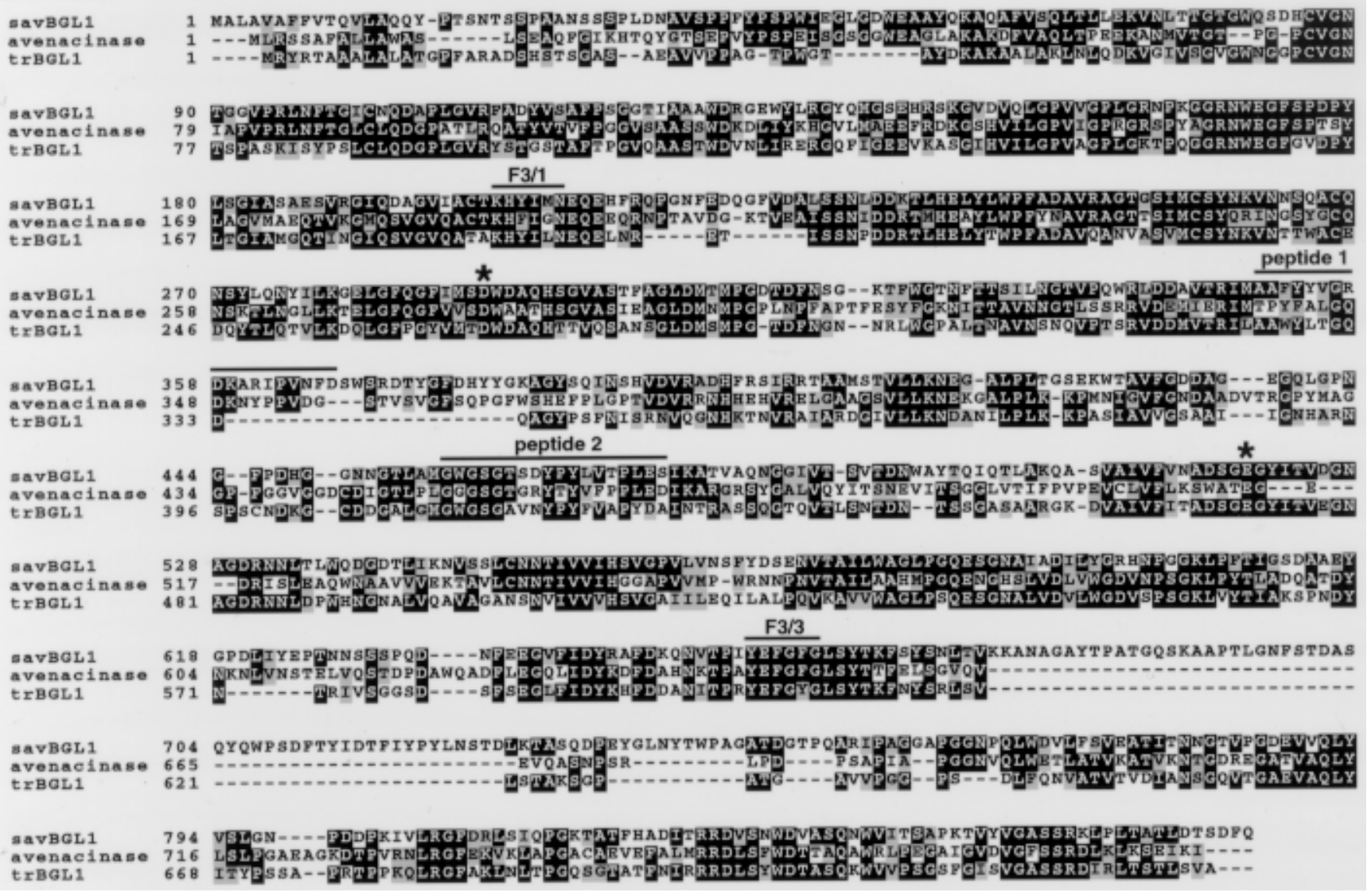

Fig. 3. Alignment of savBGL1 with two other family $3 \beta$-glucosidases, avenacinase from Gaeumannomyces graminis (U35463) and trBGL1 from Trichoderma reesei (U09580). The aspartate and glutamate that are predicted to be the catalytic nucleophile and catalytic proton donor, respectively, are marked with asterisks. The two peptides that were identified by protein sequencing are indicated, as are the two conserved peptide sequences that were used to design the degenerate primers F3/1 and F3/3. The sequences were aligned using ClustalW (Thompson et al. 1994). Identical amino acids are shaded black and similar amino acids are shaded gray. 
Although savBGL1 (AJ276675) does fall in the fungal $\beta$ glucosidase subgroup, it does not cluster tightly with the other saponin-hydrolyzing enzymes (Fig. 4). To compare the specificity of the enzymes, preparations of each were incubated with avenacin, $\alpha$-tomatine, and de-rhamnosyl26-DGA-B (Fig. 5). The activity of each enzyme was specific for its own substrate, and in this experiment no cross activity was detected after $6 \mathrm{~h}$ of incubation. After longer incubation $(24 \mathrm{~h})$, avenacinase displayed weak activity toward $\alpha$-tomatine, but the activity was considerably less than that shown toward avenacin (data not shown).

Expression of savBGL1 in planta.

An RT-PCR approach was used to detect the presence of savBGL1 mRNA. Based on information from the genomic sequence, primers were designed that would amplify bands of different lengths from genomic DNA and from cDNA. $S$. avenae was grown in liquid culture, and RNA was prepared to be used as a template for cDNA synthesis. A primer (SAV16) that hybridized to mRNA in the final exon was used to prime cDNA synthesis. PCR using this cDNA as a template, and primers that would amplify DNA between the first and the third exon (SAV15 and SAV09) generated a DNA fragment of 363 nt. This fragment was sequenced and was confirmed to be derived from savBGL1 (data not shown). PCR with genomic DNA as a template using these primers amplified a band of 703 nt that contained the first two intronic sequences. Expression of savBGL1 mRNA during infection of oat leaves was then investigated. Detached oat leaves were inoculated with a spore suspension of $S$. avenae isolate WAC1293. Following infection and symptom development, RNA was recov-

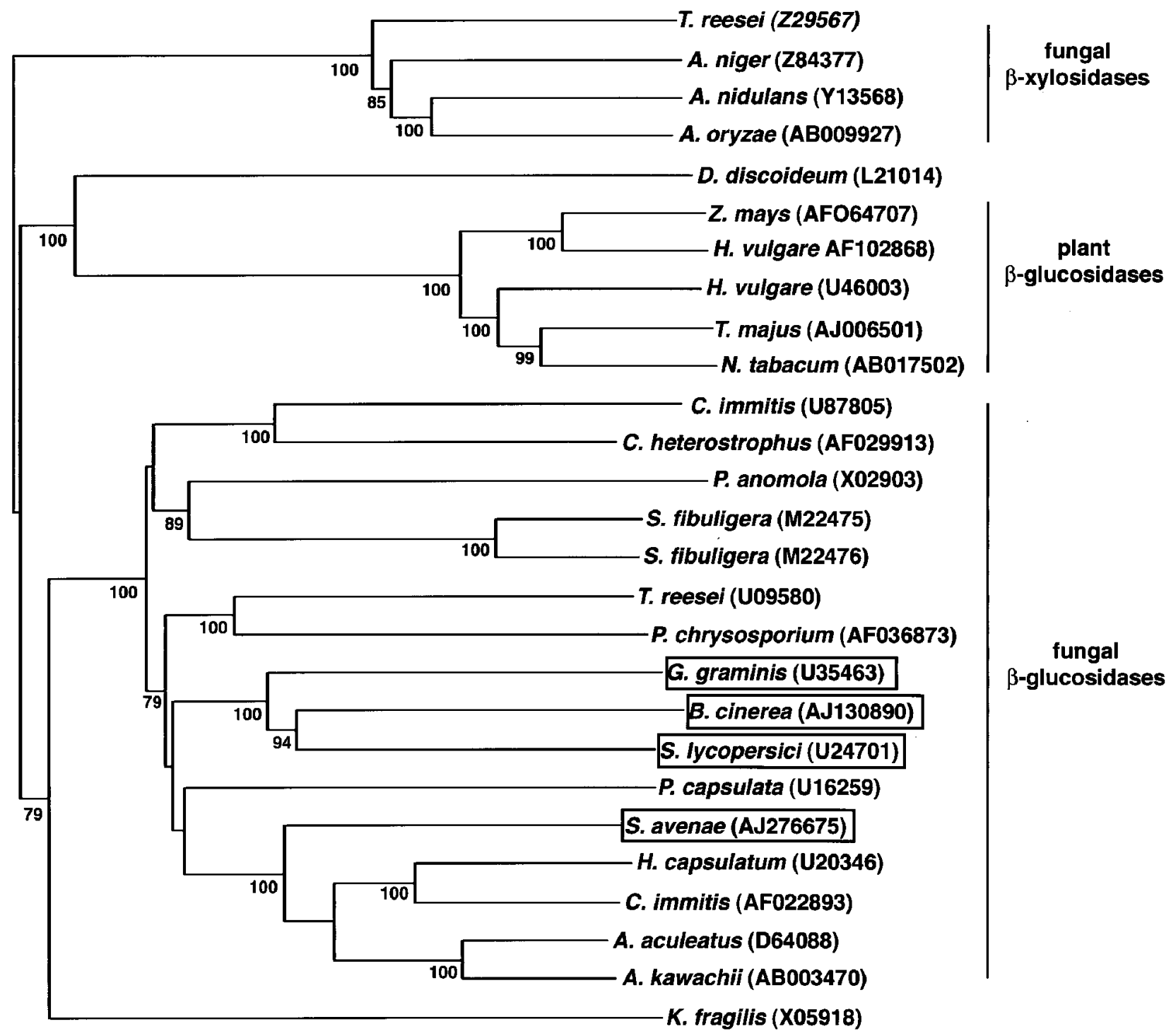

Fig. 4. Phenogram of eukaryotic family $3 \beta$-glycosidases. Complete amino acid sequences of characterized eukaryotic family $3 \beta$-glycosidases were aligned using ClustalW and the alignment used to generate this tree of relatedness (drawn using NJPlot). ClustalW and NJPlot are part of the ClustalX package (Thompson et al. 1997). Bootstrap values indicating the percentage of 1,000 trials that proteins clustered together are shown when above $70 \%$. Three distinct subgroups (fungal $\beta$-xylosidases, plant $\beta$-glucosidases, and fungal $\beta$-glucosidases) are indicated. Enzymes that have been shown to hydrolyze saponins are boxed. 
ered from infected and control leaves. cDNA synthesis using SAV16 followed by PCR using primers SAV15 and SAV09 amplified a band of 363 bp from leaves infected with wild-type fungus but not from mock-inoculated leaves (data not shown). This DNA fragment was of identical size to that amplified from savBGL1 cDNA, indicating that $s a v B G L 1$ is expressed during infection.

\section{Construction of a mutant lacking savBGL1.}

Southern blot analysis indicated that a single gene encoded savBGL1 (data not shown). To investigate the role of the protein, a mutant strain specifically disrupted in the savBGL1 gene was constructed. A disruption vector was made in which an NcoI fragment encoding the first 457 aa of savBGL1, including the predicted catalytic aspartate, was replaced by the bacterial hph gene encoding hygromycin B resistance (Fig. 6A). This vector was linearized with HindIII and used to transform S. avenae isolate WAC1293. Out of 400 hygromycin B-resistant transformants, PCR analysis using a primer in the $h p h$ sequence and a primer in the genomic sequence flanking the $s a v B G L 1$ gene identified four candidate disruptants (data not shown). These strains were further analyzed by Southern blotting, and a

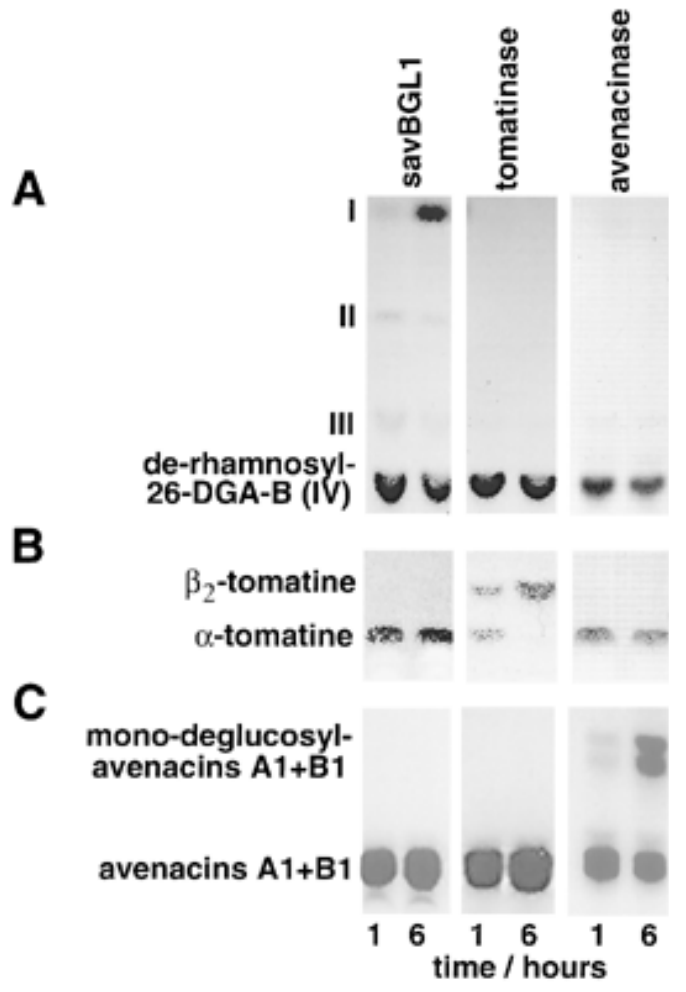

Fig. 5. Substrate specificity of family 3 saponinases. Partially purified savBGL1, tomatinase, or avenacinase enzymes were incubated with saponin substrates for 1 and $6 \mathrm{~h}$. A, Purified 26-desglucoavenacoside-BL-rhamnose (species IV) and the products of hydrolysis by savBGL1 were separated by thin-layer chromatography (TLC) and visualized by spraying the TLC plates with a $p$-anisaldehyde-sulphuric acid-acetic acid mix. $\mathbf{B}$, Pure $\alpha$-tomatine and the product of hydrolysis by tomatinase, $\beta_{2}$ tomatine, were run and visualized in the same manner. $\mathbf{C}$, Purified avenacin mixture contained all four avenacins, two of which, avenacins A-1 and B-1, were visualized under UV light. The products of hydrolysis by avenacinase (mono-deglucosyl avenacins A-1 and B-1) were also visible under UV light. single strain that contained a precise gene replacement at the savBGL1 locus was identified. Genomic DNA was digested with $X h o I$ and hybridized with the indicated probes (Fig. 6B). Probe 1 hybridizes to the 900-bp XhoI fragment from the wild type and to a larger $4.1-\mathrm{kb}$ fragment from the transformant, consistent with the loss of the internal XhoI site. This transformant lacks the NcoI region of savBGL1 (probe 2) and contains the hph gene (probe 3).

As a further test of the gene disruption, the protein compositions of the wild type and mutant were compared by two-dimensional (2-D) gel electrophoresis. The wild-type and mutant strains were grown in liquid medium, and proteins were concentrated from the culture filtrate. The proteins were run in parallel on a 2-D gel system, with separation in the first dimension based on charge and separation in the second dimension based on molecular mass. The gels were silver-stained and the patterns of the wild type and mutant compared (Fig. 7). The protein pat-

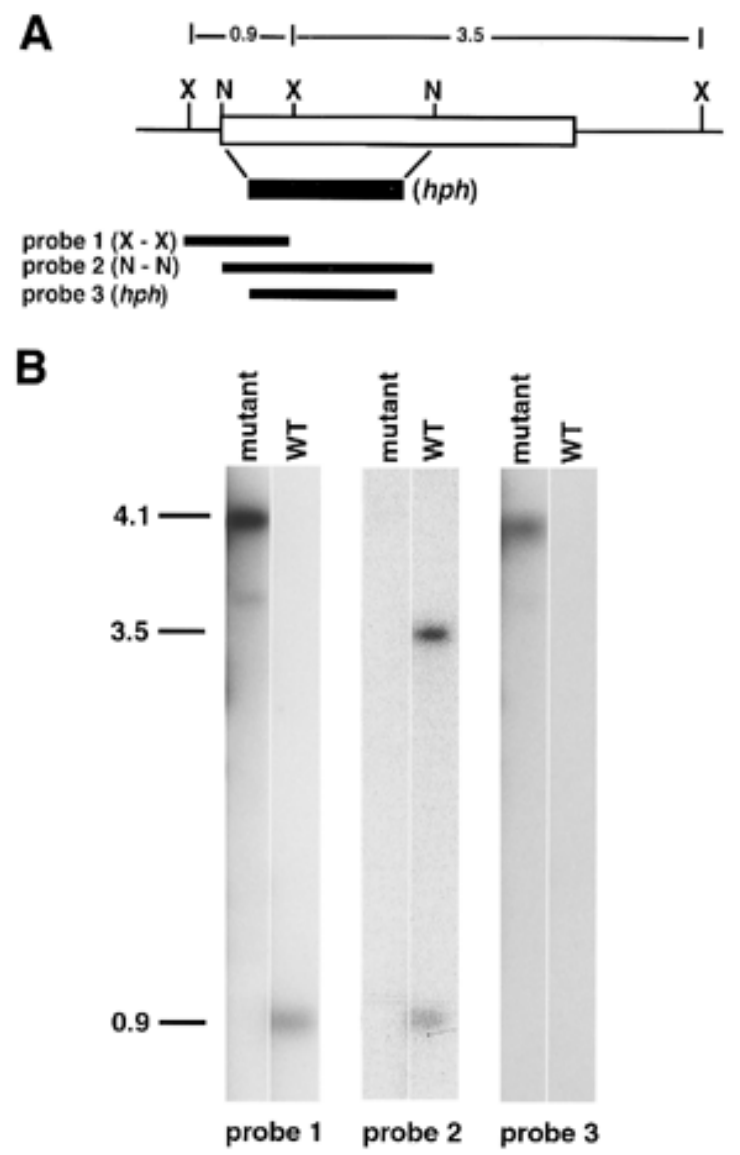

Fig. 6. Disruption of the $\operatorname{savBGL1}$ gene. A, Schematic representation of the disruption strategy. The savBGL1 coding sequence is depicted as an open box and relevant restriction enzyme sites are marked: $\mathrm{X}=\mathrm{XhoI}$ and $\mathrm{N}=N c o$ I. The NcoI fragment was replaced by the bacterial hph gene, depicted as a black box, and the linearized construct was used to transform Stagonospora avenae isolate WAC1293. The three probes used to identify correct transformants are also indicated. Probe 1 is an XhoIXhoI fragment from wild-type DNA, probe 2 is an NcoI-NcoI fragment from wild-type DNA, and probe 3 is the bacterial $h p h$ gene. B, Southern analysis of a correct integrant, labeled as mutant. Genomic DNA from the wild type and the transformant was digested with XhoI, separated by electrophoresis, transferred to a membrane by Southern blotting, and hybridized with labeled probes. 
terns were very similar, except for the absence of a single spot in the mutant protein preparation, which corresponded to the position of purified savBGL1 when run under identical conditions (Fig. 7, arrow).

\section{Phenotypic analysis of the mutant strain lacking savBGL1.}

The wild-type and mutant strains were analyzed in parallel to determine whether they differed in growth rate, extracellular protein production, resistance to avenacosides, or pathogenicity to oats. No differences in growth rates were apparent when the wild type and mutant were grown on plates using a variety of carbon sources (data not shown). The avenacoside-hydrolyzing activities present in crude filtrates from wild-type and mutant cultures were also compared. The 26-DGA-B enzyme was incubated with proteins concentrated from culture filtrate and aliquots were assayed at the times indicated (Fig. 8). Using proteins concentrated from a wild-type culture, 26-DGA-B was completely hydrolyzed by the $\alpha$-rhamnosidase to species IV after 15 to $30 \mathrm{~min}$ of incubation (Fig. 8A, lanes 3 and 4). Species IV was sequentially hydrolyzed by savBGL1 and savBGL2 to produce species III, II, and I. Species I, lacking all sugar residues, was easily detectable after $60 \mathrm{~min}$ of incubation (Fig. 8A, lane 5) and, by $240 \mathrm{~min}$, almost all the saponin had been converted to this form (Fig. 8A, lane 8). These data contrast with those for the mutant (Fig. 8B). Again, the $\alpha$-rhamnosidase rapidly converted 26-DGA-B to species IV, with complete conversion after 15 min (Fig. 8B, lane 3 ). In this case, however, conversion of species IV to species III was slow and, even after long incubation, only a proportion was converted. These data are consistent with the absence of savBGL1 from the filtrate. Further analysis using the fractionation conditions described in Figure 2 substantiated this interpretation and confirmed that the residual $\beta$ glucosidase activity is that of savBGL2 (data not shown).

Previously, it was demonstrated that 26-DGAs are considerably more toxic to wheat-attacking Stagonospora spp. than to $S$. avenae. The toxicity of the products of the avenacosidehydrolyzing enzymes, species IV to I, was also tested and it was found that, at comparable concentration, all were nontoxic to fungi (Wubben et al. 1996). The sensitivity of the wild-type and mutant strains to 26-DGAs on plates was compared. Whereas sensitive Stagonospora isolates showed 50\%

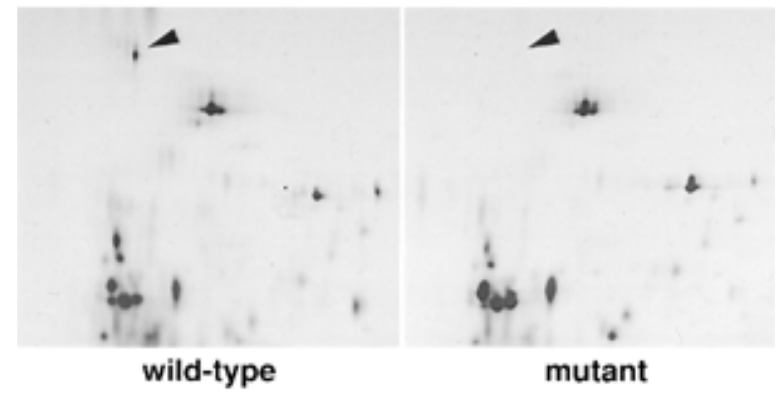

Fig. 7. Two-dimensional (2-D) gel analysis of proteins from wild-type and mutant strains. Wild-type isolate WAC1293 and mutant isolate lacking savBGL1 were grown in liquid medium. Proteins were concentrated from culture filtrates and run on 2-D gels. The regions of each gel that contained the high-molecular-weight proteins are shown for each strain. The arrow indicates the savBGL1 protein, present in the wild-type preparation but absent in the mutant one. growth inhibition at a concentration of $10 \mu \mathrm{g} / \mathrm{ml}$, neither the wild type nor the mutant displayed any growth inhibition, even at a concentration of $100 \mu \mathrm{g} / \mathrm{ml}$ (data not shown), which is well in excess of the avenacoside concentration in oat leaves (Kesselmeier and Urban 1983; Urban et al. 1983).

Tests were carried out to investigate whether the mutant lacking savBGL1 was reduced in its ability to infect oat leaves. Spores from wild-type and mutant strains were inoculated onto detached oat leaves $(3 \mu 1$ of suspension of spores at $10^{6} \mathrm{ml}^{-1}$ ) and disease lesions compared (Fig. 9). No gross differences between wild-type and mutant strains
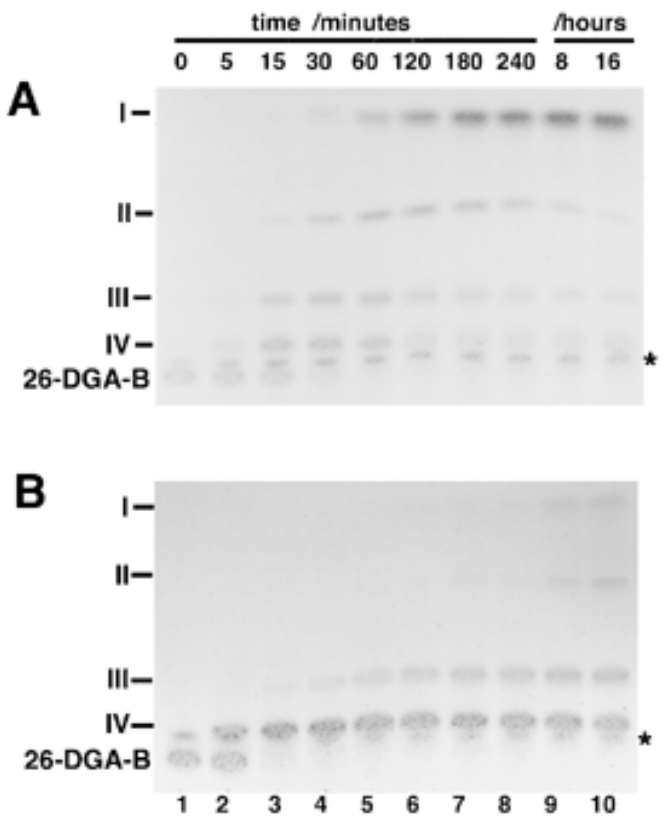

Fig. 8. Comparison of the avenacoside-hydrolyzing activities produced the wild-type and mutant Stagonospora avenae isolates. The wild-type and mutant isolates were grown in parallel under identical conditions and proteins were concentrated from culture filtrates. Equivalent amounts of proteins were incubated with 26-desglucoavenacoside (26-DGA)-B and the reactions stopped at fixed time-points. Reaction mixes were spotted onto silica plates and substrates and products resolved by thin-layer chromatography. 26-DGA-B is hydrolyzed by an $\alpha$-rhamnosidase to produce species IV, which is then sequentially hydrolyzed by $\beta$-glucosidase activity, yielding species III, II, and I (Fig. 1). The substrate and products of hydrolysis are labeled in the figure. The contaminant that is present in the substrate solution, marked with an asterisk, is not hydrolyzed and does not interfere with the reaction. Results of $\mathbf{A}$, analysis of proteins from the wild-type strain and $\mathbf{B}$, analysis from the mutant.

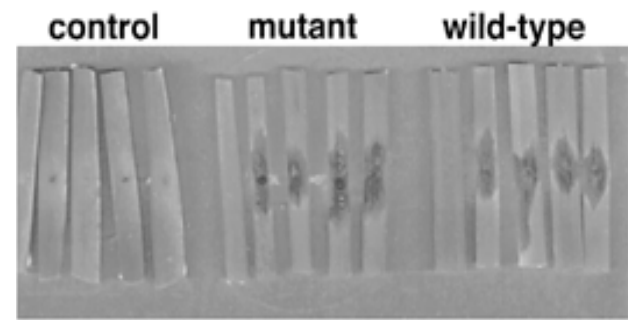

Fig. 9. Wild-type and mutant strains are both pathogenic to oat leaves. Detached oat leaves were inoculated with spore suspensions of the wild type, of the savBGL1 mutant, and of an isolate (S210/80) that is not pathogenic to oats, as a negative control. In each case, four leaves were infected and the fifth leaf is a mock-infected control. Photographs were taken 5 days after inoculation. 
were detected, and measurement of lesion lengths did not reveal significant differences between the strains. Incubation of the leaves was continued until fungal pycnidia were produced; once again, no differences between the wild type and mutant were seen. The pathogenicity assay was also carried out using low inoculum concentrations $(3 \mu \mathrm{l}$ of suspension of spores at $10^{4} \mathrm{ml}^{-1}$ ), and both strains behaved similarly under these conditions (data not shown). RT-PCR analysis confirmed that leaves infected with the mutant had no detectable levels of savBGL1 mRNA (data not shown). These data demonstrate that production of savBGL1 is not an absolute requirement for infection.

\section{DISCUSSION}

The foliar pathogen $S$. avenae secretes multiple enzymes that can utilize oat saponins as substrates. For the experiments presented in this report, 26-DGA-B was used as the enzymatic substrate, but 26-DGA-A and nonactivated avenacosides served equally well (data not shown). In liquid culture, one $\alpha$-rhamnosidase and two $\beta$-glucosidase enzymes that hydrolyze avenacosides were produced (Fig. 2 ). The production of saponin-hydrolyzing enzymes by a number of fungi has been described (Carter et al. 1999; Morrissey and Osbourn 1999; Sandrock and VanEtten 1998). Some of these enzymes have been characterized; $G$. graminis (Bowyer et al. 1995; Osbourn et al. 1991), $S$. lycopersici (Osbourn et al. 1995; Sandrock et al. 1995), Fusarium oxysporum f. sp. lycopersici (Roldan-Arjona et al. 1999), and F. solani (Lairini and Ruiz-Rubio 1998) all appear to produce single enzymes that hydrolyze sugars from the saponins present in their host plants. Isolates of B. cinerea (Quidde et al. 1998, 1999) and Gibberella pulicaris (Becker and Weltring 1998; Weltring et al. 1997, 1998) secrete multiple enzymes, each reported to act on different saponin substrates. The tomato pathogen Alternaria solani can hydrolyze several sugars from $\alpha$-tomatine, suggesting that more than one enzyme may be involved (Sandrock and VanEtten 1998; Schlösser 1975), but no enzymes from this fungus have been characterized. The work reported here is the first clear demonstration of the synthesis by a fungus of different enzymes that can act on one saponin. In culture, a single $\alpha$-rhamnosidase, the activity of which is sufficient to convert 26-DGAs to less toxic molecules, is produced by $S$. avenae WAC1293 (Fig. $2 \mathrm{~A})$. This fungus also secretes two independent $\beta$-glucosidases that can subsequently hydrolyze D-glucose residues from the saponins (Fig. 2B). Under the growth conditions employed in this study, savBGL1 was the major $\beta$-glucosidase produced. This enzyme was purified, the gene encoding the protein cloned, and the possible role of the $\beta$ glucosidase in pathogenicity investigated.

Analysis of the deduced amino acid sequence of savBGL1 shows that the protein is a member of a large class of proteins known as family $3 \beta$-glycosidases (Figs. 3 and 4). Three saponin-hydrolyzing enzymes in this family have previously been characterized. The deduced amino acid sequences of these enzymes, G. graminis avenacinase, $S$. lycopersici tomatinase, and $B$. cinerea sap1, are very similar, suggesting that saponin-hydrolyzing enzymes might form a single subgroup within family 3; however,
savBGL1 falls into a different subgroup (Fig. 4). For savBGL1 and for the other saponin-hydrolyzing enzymes, the groupings are supported by $100 \%$ bootstrap values, indicating that the separation is likely to be significant (Fig. 4). Family $3 \beta$-glycosidases contains enzymes that hydrolyze $\beta$-D-glucose or $\beta$-L-xylose residues from a wide variety of substrates. The substrate specificities of some of these enzymes have been characterized. For example, the $\beta$-glucosidases from Phanerochaete chrysosporium is involved in cellulose degradation (Li and Renganathan 1998; Lymar et al. 1995); exoglucanases from Hordeum vulgare (barley) act on plant glucans (Hrmova and Fincher 1997); and $\beta$-glucosidases from G. graminis, S. lycopersici, and B. cinerea hydrolyze plant saponins (Osbourn et al. 1995; Quidde et al. 1999; Sandrock et al. 1995). Despite the high degree of conservation among these enzymes, they can be very specific for particular substrates. Both G. graminis avenacinase and $S$. lycopersici tomatinase hydrolyze terminal $\beta$ (1-2) linked D-glucose residues from the branched sugar chains of avenacins and $\alpha$-tomatine, respectively, but have very little cross activity or activity against structurally similar molecules (Osbourn et al. 1995; Sandrock et al. 1995). Similarly, neither are active against avenacosides, and savBGL1, though active against avenacosides, had no detectable activity when tested with avenacin or $\alpha$ tomatine as a substrate (Fig. 5). Whether the substrate specificities of these enzymes is generated by the local structure of the saponin sugar chain, by the nature of the saponin backbone, or by a combination of these has yet to be determined.

Although savBGL1 is active against avenacosides, the biological significance of this activity is unknown. To address the role of savBGL1, a targeted gene replacement of savBGL1 was performed. Although only one precise replacement mutant was obtained, no differences between this strain and the wild type in growth rate under a range of conditions were observed. In addition, other than the loss of savBGL1, extracellular protein profiles and enzyme activities were similar for the wild-type and mutant strains, and pathogenicity was unaffected. In combination, these data indicate that no extraneous mutations that influenced the results were introduced into the mutant strain. Disruption of the savBGL1 gene did not cause loss of pathogenicity (Fig. 9); therefore, although the gene is expressed during infection, savBGL1 is not required for pathogenicity. The enzyme may play a subtle role during infection, perhaps contributing to overall fitness or to survival of the fungus. Whatever the role of savBGL1, it is clear that it is not required for the initial detoxification of 26-DGAs. A previous study suggested that savBGL1 may have both $\alpha$-rhamnosidase and $\beta$-glucosidase activities, but the refined assays used in the current study allowed us to refute this hypothesis. The earlier ambiguity has now been resolved and it is established that, at least in culture, a single $\alpha$-rhamnosidase is produced that detoxifies the 26-DGAs. This $\alpha$-rhamnosidase is a prime candidate as a determinant of saponin resistance, and possibly also of pathogenicity, and future work will focus on this enzyme. An interesting idea is that the avenacoside-hydrolyzing enzymes might work in concert to aid the fungus during its infection cycle. For example, the $\alpha$-rhamnosidase could be 
responsible for neutralization of the toxic saponins, whereas the $\beta$-glucosidase might then hydrolyze the neutralized saponins, generating hydrolysis products that could be used as a carbon source (VanEtten et al. 1995). Cloning of the gene encoding the $\alpha$-rhamnosidase will allow the contribution of the $\alpha$-rhamnosidase enzyme to resistance to 26-DGAs and to pathogenicity to be tested.

\section{MATERIALS AND METHODS}

Fungal isolates and growth conditions.

The isolates used in this work were previously described in Wubben et al. (1996). Isolate WAC1293 was named Septoria avenae f. sp. avenae. Revised nomenclature is now used and this isolate is named Stagonospora avenae $\mathrm{f}$. sp. avenaria (Cunfer and Ueng 1999; Farr et al. 1989). Isolate $\mathrm{S} 210 / 80$ is $S$. nodorum (Berk.) Castellani \& Germano (Castellani and Germano 1977). The isolates were stored on potato dextrose agar under mineral oil at $4{ }^{\circ} \mathrm{C}$ and were grown on Czapek Dox V-8 juice agar (Cooke and Jones $1970)$ at $20^{\circ} \mathrm{C}$. The isolates were induced to sporulate by transferring 7-day-old colonies from the dark to near-UV light with a dark and light cycle of 8 and $16 \mathrm{~h}$, respectively. Spores were harvested by flooding the plate with water and scraping with a glass rod. Concentrations of macroconidia were determined by counting using a haemocytometer.

\section{Separation of avenacoside-hydrolyzing activities from fungal culture filtrates.}

To generate culture filtrate for analysis, 2-liter conical flasks, each containing $500 \mathrm{ml}$ of Jermyn's medium (Jermyn 1959), were inoculated with spores (macroconidia) of $S$. avenae isolates to a final concentration of $5 \times 10^{7} \mathrm{liter}^{-1}$. Cultures were grown for 5 to 7 days at $22^{\circ} \mathrm{C}$ with shaking at $120 \mathrm{rpm}$. The filtrate was harvested by filtration through Miracloth (Calbiochem, San Diego, CA, U.S.A.), chilled to $4{ }^{\circ} \mathrm{C}$, and incubated for $30 \mathrm{~min}$ with a protease inhibitor cocktail. Final concentrations of inhibitors were $2 \mathrm{mM}$ EDTA, $50 \mu \mathrm{M}$ phenylmethylsulphonylfluoride, $1 \mu \mathrm{M}$ benzamidine $\mathrm{HCl}, 0.5 \mu \mathrm{M}$ phenanthroline, $0.5 \mu \mathrm{M}$ aprotinin, $2 \mu \mathrm{M}$ leupeptin, and $1.5 \mu \mathrm{M}$ pepstatin A (all from SigmaAldrich, St Louis, MO, U.S.A.). Proteins were concentrated from the filtrate by ammonium sulfate precipitation (60\% saturation), resuspended in water, and dialyzed against $20 \mathrm{mM}$ diethanolamine (DEA), $\mathrm{pH}$ 8.5. Proteins were fractionated by anion-exchange chromatography on a $4.6 \times 100-\mathrm{mm}$ (diameter $\times$ length) polyethylethylketone (PEEK) column containing Poros 20 HQ resin (PE Biosystems, Warrington, U.K.). Proteins to be fractionated were concentrated to 100 to $500 \mu \mathrm{l}$ on a Centricon C-30 column (Millipore, Bedford, MA, U.S.A.) before loading onto the anion-exchange column. The column was then washed with 10 column volumes of $20 \mathrm{mM}$ DEA, $\mathrm{pH} 8.5$, and proteins were eluted with a salt gradient of 0 to $0.6 \mathrm{M}$ $\mathrm{NaCl}$ in $20 \mathrm{mM}$ DEA, $\mathrm{pH} 8.5$, with a flow rate of $7 \mathrm{ml}$ $\mathrm{min}^{-1}$. Fractions $(1 \mathrm{ml})$ were collected and analyzed for enzymatic activity as described below.

\section{Enzyme assays.}

To assay for saponin-hydrolyzing activity, crude protein preparations or eluted fractions were incubated with $10 \mu \mathrm{g}$ of substrate in $20 \mathrm{mM}$ phosphate buffer, $\mathrm{pH} 6.2$, in a reaction volume of $100 \mu 1$. Incubations were carried out at $37^{\circ} \mathrm{C}$. To assay for $\alpha$-rhamnosidase activity, 26-DGA-B, produced as described by Wubben et al. (1996), was used as a substrate. In cases in which further hydrolysis of the product of $\alpha$-rhamnosidase was undesirable, deoxynojirimycin (Sigma-Aldrich) was included in the reaction mix at a concentration of $1.5 \mathrm{mM}$. To assay for $\beta$-glucosidase activity, de-rhamnosyl-26-DGA-B (species IV) was used as the substrate. This was generated by incubation of 26DGA-B with the crude $S$. avenae protein preparation in the presence of deoxynojirimycin. The reaction was performed to completion and the reaction mixture was freeze-dried and resuspended in $50 \%$ methanol. The solution was applied to a C-18 Sep-Pak column (Waters, Milford, MA, U.S.A.) and eluted under gravity flow with increasing concentrations of methanol. Species IV eluted in $75 \%$ methanol. Reaction conditions for $\beta$-glucosidase assays were the same as for $\alpha$-rhamnosidase, except that incubation times were 1 to $2 \mathrm{~h}$. For both assays, the reactions were stopped by freezing in liquid nitrogen and the samples were lyophilized. The lyophilized samples were extracted with methanol and spotted onto silica plates (silica G-60, 0.25 mm; Merck AG, Damstadt, Germany) for analysis by TLC. The TLC plates were developed with chloroform/ methanol/water (70:35:5.5) and then stained by spraying with a $p$-anisaldehyde/sulphuric acid/acetic acid solution (1:1:48) with baking at $170^{\circ} \mathrm{C}$ for $3 \mathrm{~min}$.

To investigate substrate specificity of family 3 saponinases, a number of saponins were incubated with different saponins. De-rhamnosyl-26-DGA-B (species IV) was prepared as described above. $\alpha$-Tomatine was purchased from Sigma-Aldrich and prepared as previously described (Osbourn et al. 1995). Avenacins were previously purified from oat roots (Carter et al. 1999). The sources of the partially purified enzymes were as follows: avenacinase (Osbourn et al. 1991), tomatinase (Osbourn et al. 1995), and savBGL1 (above). Reaction conditions using savBGL1 are described above. Reactions with avenacinase and tomatinase used $20 \mu \mathrm{g}$ of substrate and were carried out in $50 \mathrm{mM}$ sodium acetate buffer, $\mathrm{pH} 5.0$, at $37^{\circ} \mathrm{C}$.

\section{Cloning savBGL1.}

Purification of savBGL1 has already been described (Wubben et al. 1996). The purified protein was digested with cyanogen bromide as follows. Protein $(100 \mu \mathrm{g})$ was lyophilized and resuspended in $20 \mu \mathrm{l}$ of $70 \%$ formic acid. One crystal of cyanogen bromide was added and the reaction mix incubated in the dark under argon for $24 \mathrm{~h}$. Water $(200 \mu \mathrm{l})$ was added and the mixture lyophilized. The pellet was resuspended in water and lyophilized a second time. The mixture was run on a sodium dodecyl sulfate-polyacrylamide gel electrophoresis (SDS-PAGE) gel and transferred to a Problott membrane (PE Biosystems). A number of proteolytic fragments were visible and sequence information was obtained from two of them by P. Barker, Institute of Animal Physiology and Genetic Research, Cambridge Research Station, Babraham, Cambridge, U.K. This sequence information was used to design degenerate primers SAV01 (forward: 5'-ATG GCT/C/G GCT/C/A TTT/C TAT/C TAT/C GT-3') and SAV02 (reverse: 5'-GAA/G GTA/G CCA/G 
GAA/T/C CCC CAA/G/C CCC AT-3'). Peptide sequences indicated that savBGL1 was a family $3 \beta$-glycosidase; therefore, it was possible to design two more degenerate primers $5^{\prime}$ and $3^{\prime}$ of SAV01 and SAV02, respectively, that would allow the use of a nested PCR cloning strategy. These primers, F3/1 (forward: 5'-AAG CAC TT/AT/C ATC G/CG/TC AAT/C GA-3') and F3/3 (reverse: $5^{\prime}$-ATG/A CTC AAA/G CCG AT/AA/G CCC/G/A/T GA-3'), were designed using the conserved regions indicated in Figure 4. Nested PCR using primers $F 3 / 1$ and $F 3 / 3$ followed by primers SAV01 and SAV02 resulted in amplification of a 350-bp band. Amplification using SAV01 and F3/3 generated a 950-bp band. The PCR products were sequenced and found to contain overlapping nucleotide sequences. The deduced amino acid sequence contained both peptide sequences and was clearly a fragment of a family $3 \beta$-glycosidase enzyme.

The gene encoding savBGL1 was cloned from a genomic library of $S$. avenae isolate WAC1293. This library was constructed in the $\lambda$-ZAPII vector, following the manufacturer's instructions (Stratagene, La Jolla, CA, U.S.A.). The 950-bp PCR fragment was used as a probe to screen $1.5 \times 10^{5}$ recombinant plaques resulting in the isolation of 30 hybridizing phages. Phagemids of 10 hybridizing phages were recovered and further analyzed by restriction mapping. All contained overlapping DNA inserts. Based on the position of the hybridizing DNA on the insert, one phagemid was selected and the insert sequenced completely. A single ORF encoding savBGL1 was identified. The genomic sequence has been deposited in the EMBL database under accession number AJ276675.

\section{Construction of a mutant lacking savBGL1.}

The following strategy was employed to construct an isolate carrying a disrupted allele of savBGL1. A 1,700-bp NcoI fragment $(0$ to 1,758$)$ was replaced in a genomic clone pSA019 (positions 2,287 to 2,942 of accession AJ276675) by a 1,400-bp hygromycin resistance cassette (Fig. 6). This $h p h$ cassette was excised from the plasmid pCB1004 (Carroll et al. 1994) using the restriction enzyme HpaI. It contained the bacterial $h p h$ gene under the control of the Aspergillus nidulans trp $\mathrm{C}$ promoter. The disruption construct was linearized with HindIII to yield a fragment. That fragment had a $0.66-\mathrm{kb}$ flanking sequence $5^{\prime}$ of $h p h$ and 1.3 $\mathrm{kb}$ of homologous sequence 3'. S. avenae isolate WAC1293 was transformed using the protoplast procedure developed for S. nodorum (Cooley et al. 1988) and hygromycin B-resistant transformants were purified by three successive transfers to fresh agar medium containing hygromycin B at $50 \mu \mathrm{g}$ $\mathrm{ml}^{-1}$. Hygromycin resistant transformants (400) were analyzed by PCR to determine whether integration had taken place at the correct locus. PCR primers SAV12 (5'-CGT GCA GAA GGG ATG GTG G-3'), which hybridizes to savBGL1 (positions 2,161 to 2,179 of accession AJ276675), and SAV14 (5'-CGT CGC ATC GAC CCT GCG-3'), which hybridizes to the $3^{\prime}$ region of the $h p h$ gene, amplified a band of the correct size $(2.2 \mathrm{~kb})$ in four transformants. These transformants were further characterized by Southern blot analysis.

\section{Mapping of introns.}

RT-PCR was used to confirm the presence and location of introns. Based on the predicted locations of the introns, three primers were designed (numbering from the genomic sequence lodged as accession AJ276675): SAV15 (forward 2,915 to 2,$933 ; 5^{\prime}$-CCTCGCCCCGGCAGTCACG-3') is at the $5^{\prime}$ end of the coding region, SAV09 (reverse 3,618 to 3,$601 ; 5^{\prime}$-GCGAACGCCCAGAGGAGC-3') is in the exon between the second and third intron, and SAV16 (reverse 3,934 to 3,917 ; 5'-GCAGGCAATGACTCCAGC-3') is in the exon downstream of the third intron. For cDNA synthesis, RNA was extracted from fungal mycelia using a Qiagen RNA extraction kit (Qiagen, Crawley, U.K.). Total RNA $(1 \mu \mathrm{g})$ was used as a template with SAV16 as the priming oligonucleotide. The reaction was carried out at $42^{\circ} \mathrm{C}$ for $45 \mathrm{~min}$ in $20 \mu \mathrm{l}$ using Expand reverse transcriptase (Boehringer Mannheim, Mannheim, Germany) and the manufacturer's buffer. For PCR, primer combinations SAV15/SAV09 and SAV15/SAV16 were used in reactions with $5 \mu \mathrm{l}$ of the cDNA reaction mix, or $5 \mu \mathrm{g}$ of chromosomal DNA, as the template. Reaction conditions for the SAV15/SAV0 9 combination were $94^{\circ} \mathrm{C}$ for $30 \mathrm{~s}, 58^{\circ} \mathrm{C}$ for $30 \mathrm{~s}$, and $72^{\circ} \mathrm{C}$ for $60 \mathrm{~s}$, cycled 35 times; and for the SAV15/SAV16 combination were $94^{\circ} \mathrm{C}$ for $30 \mathrm{~s}, 58^{\circ} \mathrm{C}$ for $30 \mathrm{~s}$, and $72^{\circ} \mathrm{C}$ for $60 \mathrm{~s}$, cycled 35 times. Using primers SAV15 and SAV09, bands of 703 and 363 bp were amplified from genomic DNA and cDNA, respectively, and, using SAV15 and SAV16, bands of 1,019 and $631 \mathrm{bp}$ were amplified from genomic DNA and cDNA, respectively. The bands of 363 and 631 bp were sequenced to map the positions of the introns. The position of the primers was such that the 363-bp fragment allowed mapping of the junctions of the first and second introns, and the 631-bp fragment allowed mapping of the junctions of all three introns.

\section{Pathogenicity assays.}

Pathogenicity assays were performed as described in Wubben et al. (1996). Briefly, leaf segments were cut from 12-day-old seedlings of hexaploid oat species Avena sativa cv. Image and placed on water agar containing benzimidazole at $150 \mathrm{mg} \mathrm{l}^{-1}$. A spore suspension $\left(3 \mu \mathrm{l}, 1 \times 10^{6} \mathrm{ml}^{-1}\right)$ in a $0.1 \%$ Tween 20 solution was inoculated onto the detached leaf sections and incubated at $20^{\circ} \mathrm{C}$ with $16 \mathrm{~h}$ of light until symptom development. Mock-inoculated leaf sections received $3 \mu \mathrm{l}$ of $0.1 \%$ Tween 20 solution. Lesion lengths were measured from 3 to 14 days after inoculation. Statistical analysis was carried out using Student's $t$ test.

\section{RT-PCR to detect savBGL1 mRNA in infected leaves.}

Leaves were mock-inoculated, inoculated with a spore suspension of WAC1293, or inoculated with a spore suspension of the mutant lacking savBGL1. For each test, four detached leaves were inoculated along their length with droplets of a spore suspension of spores at $1 \times 10^{6}$ $\mathrm{ml}^{-1}$ and incubated at $20^{\circ} \mathrm{C}$ as described above. The leaves were frozen and RNA was extracted using a Qiagen RNA extraction kit. The RNA was quantified by spectrophotometry and its integrity verified on a $1.2 \%$ agarose gel. Total RNA $(1 \mu \mathrm{g})$ was used in a cDNA synthesis reaction using primer SAV16 as described above. A nested PCR approach was then taken to identify $s a v B G L 1$ mRNA. It was necessary to use the nested strategy to minimize nonspecific amplification of plant family $3 \beta$-glucosidase genes. 
Reaction conditions for the first PCR reaction using the primer combination SAV15/SAV 16 were $94^{\circ} \mathrm{C}$ for $30 \mathrm{~s}$, $55^{\circ} \mathrm{C}$ for $30 \mathrm{~s}$, and $72^{\circ} \mathrm{C}$ for $60 \mathrm{~s}$, cycled 20 times. Out of $50 \mu \mathrm{l}$ of this reaction product, $0.01 \mu \mathrm{l}$ was used in the second PCR reaction with a SAV15-SAV09 primer combination. Conditions were $94^{\circ} \mathrm{C}$ for $30 \mathrm{~s}, 58^{\circ} \mathrm{C}$ for $30 \mathrm{~s}$, and $72^{\circ} \mathrm{C}$ for $60 \mathrm{~s}$, cycled 35 times. Nested PCR using SAV15/SAV16 followed by SAV15/SAV09, amplified a specific band of $363 \mathrm{bp}$. This band is derived from savBGL1 mRNA.

\section{2-D protein gels.}

Proteins were concentrated from culture filtrates by ammonium sulfate precipitation (60\% saturation) and dialyzed into $20 \mathrm{mM}$ phosphate buffer, $\mathrm{pH}$ 6.8. Proteins were then run on the LKB Pharmacia multiphore II 2-D gel electrophoresis system (LKB Pharmacia, Uppsala, Sweden). Separation in the first dimension was on an isoelectric focusing strip, $\mathrm{pH} 3$ to 10. Separation in the second dimension was on a 5 to $15 \%$ SDS-PAGE gradient gel. Running conditions for both dimensions and the procedure for silver staining the gels were as described in the manufacturer's handbook. The system runs two gels in parallel, allowing the use of identical running conditions for both protein samples.

\section{ACKNOWLEDGMENTS}

We thank Jaap Visser for valuable discussions, Des Higgins for advice on phylogenetics, Vicki Bamford for help with protein purification, and Jon Carter and Marie Dufresne for critical reading of the manuscript. This work was supported by BBSRC grant no. 83/P07880. The Sainsbury Laboratory is supported by the Gatsby Charitable Foundation.

\section{LITERATURE CITED}

Armah, C. N., Mackie, A. R., Roy, C., Price, K., Osbourn, A. E., Bowyer, P., and Ladha, S. 1999. The membrane-permeabilizing effect of avenacin A-1 involves the reorganization of bilayer cholesterol. Biophys. J. 76:281-290.

Becker, P., and Weltring, K. M. 1998. Purification and characterization of alpha-chaconinase of Gibberella pulicaris. FEMS Microbiol. Lett. 167:197-202.

Bowyer, P., Clarke, B. R., Lunness, P., Daniels, M. J., and Osbourn, A. E. 1995. Host range of a plant pathogenic fungus determined by a saponin detoxifying enzyme. Science 267:371-374.

Carroll, A. M., Sweigard, J. A., and Valent, B. 1994. Improved vectors for selecting resistance to hygromycin. Fungal Genet. Newsl. 41:22-23.

Carter, J. P., Spink, J., Cannon, P. F., Daniels, M. J., and Osbourn, A. E. 1999. Isolation, characterization, and avenacin sensitivity of a diverse collection of cereal-root-colonizing fungi. Appl. Environ. Microbiol. 65:3364-3372.

Castellani, E., and Germano, G. 1977. Le Stagonosporae graminicole. Ann. Fac. Sci. Agric. Univ. Torino 10:1-132.

Cooke, B. M., and Jones, D. G. 1970. The effect of near-ultraviolet radiation and agar medium on the sporulation of Septoria nodorum and S. tritici. Trans. Br. Mycol. Soc. 54:221-226.

Cooley, R. N., Shaw, R. K., Franklin, F. C. H., and Caten, C. E. 1988. Transformation of the phytopathogenic fungus Septoria nodorum to hygromycin B resistance. Curr. Genet. 13:383-389.

Cunfer, B. M., and Ueng, P. P. 1999. Taxonomy and identification of Septoria and Stagonospora species on small-grain cereals. Annu. Rev. Phytopathol. 37:267-284.

Farr, D. F., Bills, G. F., Chamuris, G. P., and Rossman, A. Y. 1989. Fungi on Plants and Plant Products in the United States. The American Phytopathological Society, St. Paul, MN.
Fukuhara, K., and Kubo, I. 1991. Isolation of steroidal glycoalkaloids from Solanum incanum by two countercurrent chromatographic methods. Phytochemistry 30:685-687.

Gus-Mayer, S., Brunner, H., Schneider-Poetsch, H. A., and Rudiger, W. 1994. Avenacosidase from oat: Purification, sequence analysis and biochemical characterization of a new member of the BGA family of $\beta$-glucosidases. Plant Mol. Biol. 26:909-921.

Hrmova, M., and Fincher, G. B. 1997. Barley $\beta$-D-glucan exohydrolases. Substrate specificity and kinetic properties. Carbohydr. Res. 305:209-221.

Jermyn, M. A. 1959. Some comparative properties of beta-glucosidases secreted by fungi. Aust. J. Biol. Sci. 12:213-222.

Kesselmeier, J., and Urban, B. 1983. Subcellular localization of saponins in green and etiolated leaves and green protoplasts of oat (Avena sativa L). Protoplasma 114:133-140.

Keukens, E. A., de Vrije, T., Fabrie, C. H., Demel, R. A., Jongen, W. M., and de Kruijff, B. 1992. Dual specificity of sterol-mediated glycoalkaloid induced membrane disruption. Biochim. Biophys. Acta 1110: 127-136.

Keukens, E. A., de Vrije, T., van den Boom, C., de Waard, P., Plasman, H. H., Thiel, F., Chupin, V., Jongen, W. M., and de Kruijff, B. 1995. Molecular basis of glycoalkaloid induced membrane disruption. Biochim. Biophys. Acta 1240:216-228.

Lairini, K., and Ruiz-Rubio, M. 1998. Detoxification of $\alpha$-tomatine by Fusarium solani. Mycol. Res. 102:1375-1380.

Li, B., and Renganathan, V. 1998. Gene cloning and characterization of a novel cellulose-binding $\beta$-glucosidase from Phanerochaete chrysosporium. Appl. Environ. Microbiol. 64:2748-2754.

Lüning, H. U., and Schlösser, E. 1976. Role of saponins in antifungal resistance VI. Interactions Avena sativa-Drechslera avenacea. J. Plant Dis. Prot. 83:317-327.

Lymar, E. S., Li, B., and Renganathan, V. 1995. Purification and characterization of a cellulose-binding $\beta$-glucosidase from cellulose-degrading cultures of Phanerochaete chrysosporium. Appl. Environ. Microbiol. 61:2976-2980.

Morrissey, J. P., and Osbourn, A. E. 1999. Fungal resistance to plant antibiotics as a mechanism of pathogenesis. Microbiol. Mol. Biol. Rev. 63:708-724.

Oleszek, W., and Jurzysta, M. 1987. The allelopathic potential of alfalfa medicagenic acid glycosides and their fate in soil environments. Plant Soil 98:67-80.

Osbourn, A. E. 1996. Preformed antimicrobial compounds and plant defense against fungal attack. Plant Cell 8:1821-1831.

Osbourn, A., Bowyer, P., Lunness, P., Clarke, B., and Daniels, M. 1995. Fungal pathogens of oat roots and tomato leaves employ closely related enzymes to detoxify different host plant saponins. Mol PlantMicrobe Interact. 8:971-978.

Osbourn, A. E., Clarke, B. R., Dow, J. M., and Daniels, M. J. 1991. Partial characterization of avenacinase from Gaeumannomyces graminis var. avenae. Physiol. Mol. Plant Pathol. 38:301-312.

Papadopoulou, K., Melton, R. E., Leggett, M., Daniels, M. J., and Osbourn, A. E. 1999. Compromised disease resistance in saponin-deficient plants. Proc. Natl. Acad. Sci. U.S.A. 96:12923-12928.

Quidde, T., Buttner, P., and Tudzynski, P. 1999. Evidence for three different specific saponin-detoxifying activities in Botrytis cinerea and cloning and functional analysis of a gene coding for a putative avenacinase. Eur. J. Plant Pathol. 105:273-283.

Quidde, T., Osbourn, A. E., and Tudzynski, P. 1998. Detoxification of $\alpha-$ tomatine by Botrytis cinerea. Physiol. Mol. Plant Pathol. 52:151-165.

Roldan-Arjona, T., Perez-Espinosa, A., and Ruiz-Rubio, M. 1999. Tomatinase from Fusarium oxysporum f. sp. lycopersici defines a new class of saponinases. Mol. Plant-Microbe Interact. 12:852-861.

Sandrock, R. W., DellaPenna, D., and VanEtten, H. D. 1995. Purification and characterization of $\beta_{2}$-tomatinase, an enzyme involved in the degradation of $\alpha$-tomatine and isolation of the gene encoding $\beta_{2}$-tomatinase from Septoria lycopersici. Mol. Plant-Microbe Interact. 8:960-970.

Sandrock, R. W., and VanEtten, H. D. 1998. Fungal sensitivity to and enzymatic degradation of the phytoanticipin $\alpha$-tomatine. Phytopathology 88:137-143.

Schlösser, E. 1975. Role of saponins in anti-fungal resistance. IV. Tomatine-dependent development of species of Alternaria on tomato fruits. Acta Phytopathol. 10:77-87.

Thompson, J. D., Gibson, T. J., Plewniak, F., Jeanmougin, F., and Higgins, D. G. 1997. The CLUSTAL X windows interface: Flexible strategies for multiple sequence alignment aided by quality analysis tools. Nu- 
cleic Acids Res. 25:4876-4882.

Thompson, J. D., Higgins, D. G., and Gibson, T. J. 1994. CLUSTAL W: Improving the sensitivity of progressive multiple sequence alignment through sequence weighting, position-specific gap penalties and weight matrix choice. Nucleic Acids Res. 22:4673-4680.

Urban, B., Laudenbach, U., and Kesselmeier, J. 1983. Saponin distribution in the etiolated leaf tissue and subcellular localization of steroidal saponins in etiolated protoplasts of oat (Avena sativa L). Protoplasma 118:121-123.

VanEtten, H. D., Sandrock, R. W., Wasmann, C. C., Soby, S. D., McCluskey, K., and Wang, P. 1995. Detoxification of phytoanticipans and phytoalexins by phytopathogenic fungi. Can. J. Bot. 73:518-525.

Varghese, J. N., Hrmova, M., and Fincher, G. B. 1999. Three-dimensional structure of a barley $\beta$-D-glucan exohydrolase, a family 3 gly- cosyl hydrolase. Struct. Fold Des. 7:179-190.

Weissenberg, M., Levy, A., Svoboda, J. A., and Ishaaya, I. 1998. The effect of some Solanum steroidal alkaloids and glycoalkaloids on larvae of the red flour beetle, Tribolium castaneum, and the tobacco hornworm, Manduca sexta. Phytochemistry 47:203-209.

Weltring, K. M., Wessels, J., and Geyer, R. 1997. Metabolism of the potato saponins $\alpha$-chaconine and $\alpha$-solanine by Gibberella pulicaris. Phytochemistry 46:1005-1009.

Weltring, K. M., Wessels, J., and Pauli, G. F. 1998. Metabolism of the tomato saponin $\alpha$-tomatine by Gibberella pulicaris. Phytochemistry 48:1321-1328.

Wubben, J. P., Price, K. R., Daniels, M. J., and Osbourn, A. E. 1996 Detoxification of oat leaf saponins by Septoria avenae. Phytopathology 86:986-992. 